\title{
ON SELECTING THE MOST RELIABLE COMPONENTS
}

\author{
DYLAN SHI \\ Department of Mathematics, Indiana University Southeast, New Albany, IN 47150, USA
}

\begin{abstract}
Consider a series system consisting of $\mathbf{n}$ components of $\mathbf{k}$ types. Whenever a unit fails, it is replaced immediately by a new one to keep the system working. Under the assumption that all the life lengths of the components are independent and exponentially distributed and that the replacement policies depend only on the present state of the system at each failure, the system may be represented by a birth and death process. The existence of the optimum replacement policies are discussed and the $\varepsilon$-optimal policies are derived. If the past experience of the system can also be utilized, the process is not a Markov process. The optimum Bayesian policies are derived and the properties of the resulting process are studied. Also, the stochastic processes are simulated and the probability of absorption, the mean time to absorption and the average proportion of the retrograde motion are approximated.
\end{abstract}

Keywords: Selection, Bayesian Decision, Absorption, Retrograde Replacement.

\section{Introduction}

Suppose we have $k$ types of components. Assume that all the life lengths of the components are independent and exponentially distributed with unknown constant failure rates $\lambda_{1}, \lambda_{2}, \cdots, \lambda_{k}$. Since the rank order of these $k$ failure rates is assumed to be completely unknown, the natural problem arises of how to select the most reliable components associated with the smallest failure rate.

The decision problem could be classified in the areas of multiple comparisons, ranking and selection and reliability theory, but especially in the area of ranking and selection. The simplest model hereby is to operate separately $k$ components, one from each type. When a component fails, we replace it immediately by a new one of the same type. The failure counting processes in time are $k$ independent Poisson processes with failure rates $\lambda_{j}, j=1,2, \cdots, k$. Dixon and Bland (1971) have observed the $k$ Poisson processes over time periods of a common fixed length and derived a Bayes solution to the problem of complete ranking through combined paired comparisons. For the same model, Goel (1972) has shown that the natural rule for selecting the population with the largest $\lambda$-value cannot be implemented in the indifference zone approach at predetermined $P^{*}$-values. Subset selection procedures, however, can be established under the $P^{*}$-criterion, as it has been done by Gupta and Huang (1975) and Gupta and Wong (1977). The alternative problem, i.e. the one concerning subset selection with emphasis on the smallest $\lambda$ value, has been treated in Gupta, Leong, and Wong (1979). Results on selecting the population with the largest $\lambda$-value in terms of the highest posterior probability of 
being the best population have been derived by Wilson (1992). Lists of the relevant literature in this area can be found in Gupta and Panchapakesan (1979) and Miescke and Shi (1995).

Recently, Miescke (1990) and Miescke and Shi (1995) have treated the problem in a new dynamic approach. Instead of observing the $k$ parallel running, independent experiments, the $k$ types of components are examined in a series operating system where it is emphasized that at each failure, an immediate replacement is made in some optimum way to keep the system running. Under this model Miescke (1990) has derived some natural 'look-ahead-one-failure' Bayes replacement policies to maximize, at each failure, the expected waiting time for the next failure in the system, or the posterior probability that the waiting time for the next failure exceeds a given value. These Bayes rules have been obtained for both cases, when the past experiences of the system could be utilized or not. In the latter case, the system can be represented by a Markov process. Miescke and Shi (1995) have studied the process for $k=2$ and, under certain conditions, derived Markov replacement policies in some optimum way to ensure that, with maximum absorption probability, the system will finally be composed of only the components of the better type, possessing the smaller failure rate.

The first objective in the present paper is to study the existence of the optimum replacement policies and define the $\varepsilon$-optimal replacement policies for the Markov process. If the past information of the system could also be utilized, the process is not a Markov process. Our second objective is to derive the optimum Bayes replacement policies through the generalized maximum likelihood method for the non-Markov processes. Finally, the absorbing property of the non-Markov processes will be studied and the results of the computer simulation of the processes will be given at the end.

\section{Policies Based only on the Present Information}

\subsection{Model Assumptions}

Consider a series system consisting of $n$ components which are all kept permanently in working condition. Each time a failure occurs, the failed component is replaced immediately by a fresh one according to some replacement policy. Available for use are two types of components. Assume that all life lengths are independent and each unit of type $k$ has a cumulative life distribution function $G_{k}(t)=1-e^{-\lambda_{k} t}, t \geq$ $0, k=0,1$, where $\lambda_{0}, \lambda_{1}>0$ are unknown parameters.

The replacement policies considered in this section depend only on the present state of the system, i.e. which type of component caused the failure and how many components of each type were in use in the system. To be more specific, let $F_{i}, 0<i \leq n\left(S_{i}, 0 \leq i<n\right)$ denote the probability of making a replacement with a component of type $0(1)$, after a component of type 1(0) fails when the system contains $i$ components of type 1 , and let $\bar{F}_{i}=1-F_{i}$ and $\bar{S}_{i}=1-S_{i}$. Assume that $F_{i}>0, S_{i}>0,0<i<n$ and $F_{n}=S_{0}=0$, i.e. the state of the system will not be 
changed if all the components in use are of the same type. Particular policies will be denoted by $a=\left(F_{1}, \cdots, F_{n} ; S_{0}, \cdots, S_{n-1}\right)$ while the set of all possible replacement policies under consideration will be denoted $\mathcal{A}$. Note that if type 1 is better than type 0 , i.e. if $\lambda_{1}<\lambda_{0}$, then $S$ and $\bar{F}$ are suggesting a desirable replacement and $\bar{S}$ and $F$ a wrong replacement.

Let $X(t)$ denote the number of units of type 1 in the system, operating at time $t$. Then, the motion of $X(t)$ can be described as follows: if, at time $t$, the process is in state $i, 0<i<n$, then, after staying at state $i$ for an exponentially distributed length of time, it may move to state $i+1$ (a unit of type 0 fails and is replaced by a new one of type 1) or state $i-1$ (a type 1 fails and is replaced by a type 0 ), or stay at state $i$ (any one type fails and is replaced by the same type). If $X(t)=0$ or $n$, then the state of the process will never be changed. Due to the lack of memory of the exponential law, the process $\{X(t), t \geq 0\}$ is a birth and death process with finite state space $\{0,1,2, \cdots, n\}$ and absorbing states 0 and $n$. (Miescke and Shi, 1995).

\subsection{The existence of optimum replacement policies}

\subsubsection{The imbedded Markov chain}

The first natural step is to find a replacement policy which maximizes the probability that the process ends up in the better of the two absorbing states, i.e. all the components in use are of the same type which has the smaller $\lambda$-value. To this end, we introduce an embedded Markov chain as follows. Let $\tau_{0}=0<\tau_{1}<\tau_{2}<$ $\cdots<\tau_{r}<\cdots$ be the successive transition times of the birth and death process, i.e. the successive moments when a component fails and is replaced immediately, according to the replacement policy, by a unit of different type. Let $X_{0}=X(0)$, $X_{r}=X\left(\tau_{r}\right), r \geq 1$. Then, $\left\{X_{r}, r \geq 0\right\}$ is an imbedded Markov chain associated with the given Markov process $\{X(t), t \geq 0\}$. This Markov chain records only the jumps of the birth and death process at the transition times, but the probabilities of absorption into states 0 or $n$ for this Markov chain are the same as for the birth and death process since both of them execute the same transitions.

Let $a_{i j}$ denote the probability that, starting at a transient state $i \in\{1,2, \cdots, n-$ $1\}$, the Markov chain ends up in an absorbing state $j \in\{0, n\}$. Then, for any replacement policy $a \in \mathcal{A}$, the absorption probabilities $a_{i j}$ can be written in the following concise form:

$$
\begin{aligned}
& a_{1 n}=\left[\sum_{i=0}^{n-1}\left(\begin{array}{c}
n-1 \\
i
\end{array}\right)^{-1} \alpha_{i} \delta^{i}\right]^{-1}, \\
& a_{i n}=a_{1 n} \sum_{j=0}^{i-1}\left(\begin{array}{c}
n-1 \\
j
\end{array}\right)^{-1} \alpha_{j} \delta^{j}, 1<i<n,
\end{aligned}
$$




$$
a_{i 0}=a_{1 n} \sum_{j=i}^{n-1}\left(\begin{array}{c}
n-1 \\
j
\end{array}\right)^{-1} \alpha_{j} \delta^{j}, 1 \leq i<n,
$$

where $\delta=\frac{\lambda_{1}}{\lambda_{0}}, \alpha_{i}=\frac{F_{1} F_{2} \cdots F_{i}}{S_{1} S_{2} \cdots S_{i}}, 0<i<n, \alpha_{0}=1$. (Miescke and Shi, 1995)

\subsubsection{The symmetric replacement policies}

Our goal is to find a replacement policy which maximizes the probability that the process ends up in the better of the two absorbing states. Since we do not know which type of components is better we should find a policy that maximizes $a_{i n}, 0<i<n$ when type 1 is better $(\delta<1)$, and simultaneously, minimizes $a_{i n}$ (maximizes $a_{i 0}$ ) when type 0 is better $(\delta>1)$. Unfortunately, the problem has no solution if the replacement policies are not symmetric or the system is not completely symmetric, i.e. if the fixed number $n$ of components in the system is odd, $n=2 m+1$, say, or $n$ is even, $n=2 m$, say, but the starting state $i$ is not $m$, i.e. the system starts with unequal numbers of components of each type.

The first statement is obvious since $0<a_{\text {in }}<1$ for all $\delta>0$ and all $a \in \mathcal{A}$, and the limit of $a_{i n}$ is $0(1)$ as $S_{n-1}\left(F_{1}\right)$ approaches 0 , that is, $\inf _{\mathcal{A}} a_{i n}=0$ and $\sup _{\mathcal{A}} a_{\text {in }}=1$ for all $\delta>0$. This indicates that there does not exist any optimum replacement policies in $\mathcal{A}$, nor even in the extended policy set $\overline{\mathcal{A}}=\left\{a \mid F_{i} \geq 0,0<\right.$ $\left.i<n, F_{n}=0 ; S_{i} \geq 0,0<i<n, S_{0}=0\right\}$. To overcome the obstacles, it is reasonable to consider only those replacement policies which treat the two types of components without any bias towards their given labels, i.e. which act analogously if the labels 'type 0' and 'type 1' are exchanged. Thus, throughout the sequel, it is assumed that the replacement policies are symmetric in the following way: $F_{i}=S_{n-i}, i=1,2, \cdots, n$. The set of all symmetric replacement policies and its extension will be denoted by $\mathcal{A}_{1}$ and $\overline{\mathcal{A}}_{1}=\left\{a \mid F_{i}=S_{n-i} \geq 0,0<i<n, F_{n}=\right.$ $\left.S_{0}=0\right\}$, respectively.

To prove the second statement and compare the replacement policies, let us consider the extreme case where $F_{1}=S_{n-1}$ approaches 0 . It follows that $\alpha_{i}$ approaches $0, i=1,2, \cdots, n-2$, and consequently, $a_{i n}$ approaches $a_{i n}^{*}=\frac{1}{1+\delta^{n-1}}$.

If the system is not completely symmetric, then $\sup _{\mathcal{A}_{1}} a_{\text {in }}$ for $\delta<1$ and inf $\mathcal{A}_{1} a_{i n}$ for $\delta>1$ are not the same. To see this, let us investigate the case where $n=2 m+1$ and $i \leq m$, for instance.

After some standard steps of calculation, the difference $a_{i n}-a_{i n}^{*}$ can be written as

$$
a_{i n}-a_{i n}^{*}=\frac{\sum_{j=0}^{i-1}\left(\begin{array}{c}
n-1 \\
j
\end{array}\right)^{-1} \alpha_{j} \delta^{n-1+j}-\sum_{j=0}^{n-1-i}\left(\begin{array}{c}
n-1 \\
j
\end{array}\right)^{-1} \alpha_{j} \delta^{n-1-j}}{\left(1+\delta^{n-1}\right) \sum_{j=0}^{n-1}\left(\begin{array}{c}
n-1 \\
j
\end{array}\right)^{-1} \alpha_{j} \delta^{j}} .
$$

Its denominator is positive, while its numerator can be written as

$$
\sum_{j=0}^{i-1}\left(\begin{array}{c}
n-1 \\
j
\end{array}\right)^{-1} \alpha_{j} \delta^{n-1-j}\left(\delta^{2 j}-1\right)-\sum_{j=i}^{n-1-i}\left(\begin{array}{c}
n-1 \\
j
\end{array}\right)^{-1} \alpha_{j} \delta^{n-1-j}
$$


which is negative if $\delta<1$. That is, $a_{i n}<a_{i n}^{*}$ for all $\delta<1$. Note that the limit of $a_{i n}$ is $a_{i n}^{*}$ as $F_{1}$ approaches 0 . It follows that, in the present case, $\sup _{\mathcal{A}_{1}} a_{i n}=a_{i n}^{*}$ for all $\delta<1$.

On the other hand, if we multiply both the numerator and the denominator of $a_{i n}$ in Eq.(1) by $S_{1} S_{2} \cdots S_{m}$, we can see that each term of the numerator and the denominator contains a common factor $S_{m}$, except for the middle term of the denominator, which is $\left(\begin{array}{c}n-1 \\ m\end{array}\right)^{-1} F_{1} F_{2} \cdots F_{m} \delta^{m}$. It follows that $a_{i n}$ approaches 0 as $S_{m}$ approaches 0 and consequently, in the present case, $\inf _{\mathcal{A}_{1}} a_{i n}=0$ for $\delta>1$. This indicates that there does not exist any optimum replacement policies in $\mathcal{A}_{1}$, nor in its extension $\overline{\mathcal{A}}_{1}$.

\subsubsection{The completely symmetric system}

For the case $n=2 m>2$ and $i=m$, due to the complete symmetry, it can be seen that if $\delta<1$, then

$$
a_{m n}^{w}<a_{m n}<a_{m n}^{*}, \text { for all } a \in \mathcal{A}_{1} ;
$$

and if $\delta>1$, then

$$
a_{m n}^{*}<a_{m n}<a_{m n}^{w}, \text { for all } a \in \mathcal{A}_{1},
$$

where $a_{m n}^{w}=\frac{1}{1+\delta}$. Since the limit of $a_{m n}$ is $a_{m n}^{*}$ as $F_{1}$ approaches 0 we see that $a_{m n}^{*}=\sup _{\mathcal{A}_{1}} a_{m n}$ for all $\delta<1$ and $a_{m n}^{*}=\inf _{\mathcal{A}_{1}} a_{m n}$ for all $\delta>1$. Analogously, as an interesting by-product, it is readily seen that $a_{m n}^{w}=\inf _{\mathcal{A}_{1}} a_{m n}$, for all $\delta<1$ and $a_{m n}^{w}=\sup _{\mathcal{A}_{1}} a_{m n}$, for all $\delta>1$ since the limit of $a_{m n}$ is $a_{m n}^{w}$ as $S_{m-1}$ approaches 0 for all $\delta>0$.

It is now obvious that there is no optimal policy even for the completely symmetric system, since the ranges of the absorption probability $a_{m n}$ are open intervals. But it can also be seen that, in this case, $\varepsilon$-optimal policies do exist.

\subsection{The $\varepsilon$-optimal policies}

A policy $a^{\varepsilon}$ is called an $\varepsilon-$ optimal replacement policy in $\mathcal{A}_{1}$ if, simultaneously,

$$
\begin{aligned}
& a_{m n}^{\varepsilon}<a_{m n}^{*}+\varepsilon \text { for all } \delta>1, \\
& a_{m n}^{\varepsilon}>a_{m n}^{*}-\varepsilon \text { for all } \delta<1 .
\end{aligned}
$$

To see the existence of the $\varepsilon$-optimal policies, let us have a further investigation of the difference $d=a_{m n}-a_{m n}^{*}$, which can be written as

$$
d=\frac{\left(\delta^{2}-1\right) \sum_{i=1}^{m-1}\left(\begin{array}{c}
n-1 \\
i
\end{array}\right)^{-1} \alpha_{i} \delta^{n-1-i} \sum_{j=0}^{i-1} \delta^{2 j}}{\left(1+\delta^{n-1}\right) \sum_{i=0}^{m-1}\left(\begin{array}{c}
n-1 \\
i
\end{array}\right)^{-1} \alpha_{i}\left(\delta^{i}+\delta^{n-1-i}\right)}
$$


The denominator of $d$ is a polynomial of $\delta$ with degree $2 n-2$ and is always greater than 1 , while the numerator is a polynomial of $\delta$ with degree $n+m-2$ and each term of the numerator contains a common factor $F_{1}$. Hence, for all $a \in \mathcal{A}_{1}, d / F_{1}$ is a continuous function of $\delta$ in $(0, \infty)$. Since $\lim _{\delta \rightarrow 0} d=0$ and $\lim _{\delta \rightarrow \infty} d=0$, we see that $d / F_{1}$ is bounded from above on $(0, \infty)$. That is, $|d| \leq F_{1} M, 0<\delta<\infty$, for some positive number $M$. This implies that $a_{m n}$ approaches $a_{m n}^{*}$ uniformly in $\delta$ as $F_{1}$ approaches zero. Consequently, the following theorem has been shown to be true.

THEOREM 1 The best replacement policy, which maximizes the probability that the process ends up in the better of the two absorbing states does not exist, but $\varepsilon$-optimal policies do exist if the system is completely symmetric.

The $\varepsilon$-optimal policies described above suggest that if the system contains only one component of type 1 (or type 0 ) and it fails, then it should be replaced by a unit of the same type with a probability as close to one as possible. The process then stops very slowly to ensure that it will end up in the better absorbing state with the absorption probability $a_{m n}$ as close to $a_{m n}^{*}$ as desired.

The remaining parts of the above policies, $F_{i}=S_{n-i}, i=2,3, \cdots, n-1$, are completely free. We could apply, for example, the Play the Winner policy, denoted by $\mathcal{P}: F_{i}=S_{i}=1, i=1,2, \cdots, n-1$, i.e. if the failure is caused by a component of type $1(0)$, then it should be replaced by a component of type $0(1)$. The probability of absorption for $\mathcal{P}$ turns out to be

$$
a_{m n}^{p}=\sum_{i=0}^{m-1}\left(\begin{array}{c}
n-1 \\
i
\end{array}\right)^{-1} \delta^{i} / \sum_{i=0}^{n-1}\left(\begin{array}{c}
n-1 \\
i
\end{array}\right)^{-1} \delta^{i} .
$$

(Miescke and Shi, 1995). Now we could recommend an $\varepsilon$-optimal policy, $\mathcal{P}_{\sigma}$ as follows: $F_{1}=S_{n-1}=\sigma$ and $F_{i}=S_{n-i}=1, i=2,3, \cdots, n-1$.

The probability of absorption into the better state for $\delta=1 / 2$ and $n=4,8,12$ are listed in Table 1 , where $\mathcal{B}(\mathcal{W})$ stands for the best (worst) limiting value $a_{m n}^{*}$ $\left(a_{m n}^{w}\right)$ and $\mathcal{P}_{0.1}$ for an $\varepsilon$-optimal policy $\mathcal{P}_{\sigma}$, with $\sigma=0.1$.

Table 1. Absorption Probabilities

\begin{tabular}{|r|cccc|}
\hline $\mathrm{n}$ & $\mathcal{B}$ & $\mathcal{P}_{0.1}$ & $\mathcal{P}$ & $\mathcal{W}$ \\
\hline 4 & 0.8889 & 0.8841 & 0.8485 & 0.6667 \\
\hline 8 & 0.9922 & 0.9918 & 0.9879 & 0.6667 \\
\hline 12 & 0.9995 & 0.9995 & 0.9993 & 0.6667 \\
\hline
\end{tabular}

\section{Policies based on both the present and the past information}

Suppose that the past information of the system is available and, from now on, that the replacement policies depend not only on the present information, but also 
on the past experience of the system. Then, the process $\{X(t), t \geq 0\}$ is not a Markov process any more. To find an optimum replacement policy, we employ the Bayesian approach.

\subsection{The likelihood}

Let $n_{1 l}\left(n_{0 l}\right), l \geq 1$ be the number of components of type $1(0)$, operating in the system between the $(l-1)$ th and $l$ th failure, $n_{11}=n_{01}=m=n / 2$. Let $I_{l}$ denote the type of component which causes the $l$ th failure, $R_{l}$ the type of component which replaced the $l$ th failure, $I_{1 l}\left(I_{0 l}\right)$ the number of failures caused by components of type 1 ( 0$)$ during the first $l$ failures and $R_{1 l}\left(R_{0 l}\right)$ the number of components of type $1(0)$, which replaced the failed units during the first $l$ failures, $l \geq 1$. Then, it is obvious that $I_{1 l}=\sum_{j=1}^{l} I_{j}, I_{0 l}=l-I_{1 l}, R_{1 l}=\sum_{j=1}^{l} R_{j}, R_{0 l}=l-R_{1 l}$ and $n_{1 l}=m-I_{1 l-1}+R_{1 l-1}, \quad n_{0 l}=m+I_{1 l-1}-R_{1 l-1}, l \geq 1$.

The probability density of $I_{l}$, given $n_{1 l}$, is

$$
f_{l}\left(k \mid \lambda, n_{1 l}\right)=\frac{n_{k l} \lambda_{k}}{n_{1 l} \lambda_{1}+n_{0 l} \lambda_{0}}, k=1,0,
$$

which depends on the unknown parameter $\lambda=\left(\lambda_{1}, \lambda_{0}\right)$, the type of components which caused the first $l-1$ failures, $I_{1}, I_{2}, \cdots, I_{l-1}$, and the type of components which made the first $l-1$ replacements, $R_{1}, R_{2}, \cdots, R_{l-1}$.

Note that a replacement policy is just a series of probability densities of $R_{l}, l \geq 1$. It is easily seen that at the first stage, the policy depends only on $I_{1}$. At the second stage, the policy depends only on $I_{1}, R_{1}$, and $I_{2}$. Since $R_{1}$ is determined by $I_{1}$, we see that, at the second stage, the policy depends only on $I_{1}$ and $I_{2}$. Thus, we conclude that, at the $l$ th stage, the replacement policy under consideration depends only on $I_{1}, I_{2}, \cdots, I_{l}$ and $\lambda$. As a consequence, we may obtain, for instance, that $P\left\{I_{2}=i_{2} \mid I_{1}=i_{1}\right\}=P\left\{I_{2}=i_{2} \mid I_{1}=i_{1}, R_{1}=r_{1}\right\}=n_{i_{2} 2} \lambda_{i_{2}} /\left(n_{12} \lambda_{1}+n_{02} \lambda_{0}\right)$, where $n_{12}=m-i_{1}+r_{1}, n_{02}=m+i_{1}-r_{1}$, and $r_{1}$ depends on $i_{1}$.

It is now readily seen that the likelihood of $I=\left(I_{1}, I_{2}, \cdots, I_{l}\right)$, with $\Delta=\lambda_{0} / \lambda_{1}$, is

$$
\begin{aligned}
\mathcal{L}=\mathcal{L}(I \mid \lambda) & =P\left\{I_{1}=i_{1}, I_{2}=i_{2}, \cdots, I_{l}=i_{l}\right\} \\
& =P\left\{I_{1}=i_{1}\right\} \cdots P\left\{I_{l}=i_{l} \mid I_{j}=i_{j}, 1 \leq j<l\right\} \\
& =\prod_{j=1}^{l} \frac{n_{i_{j} j} \lambda_{i_{j}}}{n_{1 j} \lambda_{1}+n_{0 j} \lambda_{0}} \\
& =\left(\prod_{j=1}^{l} n_{i_{j} j}\right) \frac{\lambda_{1}^{I_{1 l}} \lambda_{0}^{I_{0 l}}}{\prod_{j=1}^{l}\left(n_{1 j} \lambda_{1}+n_{0 j} \lambda_{0}\right)} \\
& =\left(\prod_{j=1}^{l} n_{i_{j} j}\right) \frac{\Delta^{I_{0 l}}}{\prod_{j=1}^{l}\left(n_{1 j}+\Delta n_{0 j}\right)} .
\end{aligned}
$$




\subsection{Bayes replacement policy}

As usual, if there is no a priori known preference between the two types of components, symmetric priors are the natural choice. Let us assume, throughout the sequel, that $\lambda=\left(\lambda_{0}, \lambda_{1}\right)$ is a realization of a random vector $\Lambda=\left(\Lambda_{0}, \Lambda_{1}\right)$ with a prior density $\pi(\lambda)$ which is permutation symmetric. Then, the joint density of $I=\left(I_{1}, I_{2}, \cdots, I_{l}\right)$ and $\Lambda$ is

$$
\mathcal{L} \pi=\left(\prod_{j=1}^{l} n_{i_{j} j}\right) \frac{\lambda_{1}^{I_{1 l}} \lambda_{0}^{I_{0 l}} \pi(\lambda)}{\prod_{j=1}^{l}\left(n_{1 j} \lambda_{1}+n_{0 j} \lambda_{0}\right)}
$$

the marginal density of $I$ is

$$
M=\int_{R_{+}^{2}} \mathcal{L} \pi d \lambda=\left(\prod_{j=1}^{l} n_{i_{j} j}\right) m_{1},
$$

where $m_{1}=\int_{R_{+}^{2}} \frac{\lambda_{1}^{I_{11}} \lambda_{0}^{I_{0 l}} \pi(\lambda)}{\prod_{j=1}^{l}\left(n_{1 j} \lambda_{1}+n_{0 j} \lambda_{0}\right)} d \lambda$, and hence, the posterior density of $\Lambda$, given $I$, is

$$
\begin{aligned}
\pi(\lambda \mid I) & =\frac{\lambda_{1}^{I_{1 l}} \lambda_{0}^{I_{0 l}} \pi(\lambda)}{\prod_{j=1}^{l}\left(n_{1 j} \lambda_{1}+n_{0 j} \lambda_{0}\right) m_{1}} \\
& =\frac{\Delta^{I_{0 l}} \pi(\lambda)}{\prod_{j=1}^{l}\left(n_{1 j}+\Delta n_{0 j}\right) m_{1}}
\end{aligned}
$$

Under $0-1$ loss, the posterior expected losses of actions $R_{l}=1$ and 0 are

$$
E^{\pi(\lambda \mid I)} L(\lambda, 1)=\int_{\left(\lambda_{1}>\lambda_{0}\right)} \frac{\Delta^{I_{0 l}} \pi(\lambda)}{\prod_{j=1}^{l}\left(n_{1 j}+\Delta n_{0 j}\right) m_{1}} d \lambda
$$

and

$$
\begin{aligned}
E^{\pi(\lambda \mid I)} L(\lambda, 0) & =\int_{\left(\lambda_{1}<\lambda_{0}\right)} \frac{\Delta^{I_{0 l}} \pi(\lambda)}{\prod_{j=1}^{l}\left(n_{1 j}+\Delta n_{0 j}\right) m_{1}} d \lambda \\
& =\int_{\left(\lambda_{1}>\lambda_{0}\right)} \frac{\Delta^{I_{1 l}} \pi(\lambda)}{\prod_{j=1}^{l}\left(\Delta n_{1 j}+n_{0 j}\right) m_{1}} d \lambda,
\end{aligned}
$$

where the last equality is obtained by exchanging $\lambda_{1}$ and $\lambda_{0}$. Hence, a sufficient condition for $E^{\pi(\lambda \mid I)} L(\lambda, 1)<E^{\pi(\lambda \mid I)} L(\lambda, 0)$ is, for all $0<\Delta<1$,

$$
\frac{\Delta^{I_{0 l}}}{\prod_{j=1}^{l}\left(n_{1 j}+\Delta n_{0 j}\right)}<\frac{\Delta^{I_{1 l}}}{\prod_{j=1}^{l}\left(\Delta n_{1 j}+n_{0 j}\right)}
$$

To summarize our results, let us define 


$$
d_{l}=d_{l}(x)=x^{I_{1 l}-I_{0 l}} \prod_{j=1}^{l} \frac{n_{1 j}+x n_{0 j}}{x n_{1 j}+n_{0 j}}, l \geq 1 .
$$

The following has been shown to be true.

THEOREM 2 Assume that $\Lambda=\left(\Lambda_{0}, \Lambda_{1}\right)$ has a permutation symmetric probability density $\pi$. Then, under $0-1$ loss, the Bayes replacement policy which minimizes the posterior expected loss is as follows: after the lth failure, replace the failed unit by a component of type $1(0)$ if $d_{l}>(<) 1$ for all $0<x<1$.

CoRollary 1 Suppose that $\Lambda=\left(\Lambda_{0}, \Lambda_{1}\right)$ has two possible values $(u, v)$ and $(v, u), u<v$, say, with both values being equally likely, i.e. $\pi(u, v)=\pi(v, u)=1 / 2$. Then, under 0-1 loss, the Bayes rule for the system is $R_{l}=1(0)$ if $d_{l}\left(\frac{u}{v}\right)>(<) 1$.

\subsection{An Example}

Example 1: Suppose that $\Lambda=\left(\Lambda_{0}, \Lambda_{1}\right)$ has two possible values $(1,2)$ and $(2,1)$ with $\pi(1,2)=\pi(2,1)=1 / 2$. Then, the Bayes rule is that $R_{l}=1(0)$ if

$$
d_{l}\left(\frac{1}{2}\right)=2^{I_{0 l}-I_{1 l}} \prod_{j=1}^{l} \frac{2 n_{1 j}+n_{0 j}}{n_{1 j}+2 n_{0 j}}>(<) 1 .
$$

Assume that $n=8$ and $I=(0,1,1,0,1,0,0,0,0,0)$, i.e. $I_{1}=0, I_{2}=1$, etc. Then, following the Bayes rule we obtain that $R=(1,1,0,1,0,1,1,1,1,1)$. The computations are very simple and hence omitted.

Remark. From the example, one sees that after the 9th replacement, all the components in use are of type 1 . Then, definitely, the next failure will be caused by a component of type 1 . According to the replacement rule, we see that the failed unit must be replaced by a component of the same type, i.e. type 1 . Hence after the next replacement, all the components in use are still of type 1 . This is always true for the successive replacements after the 9th. We conclude that the process has two absorbing states. The proof of the absorption property of the process will be given in the next section.

\section{Simulation of the process}

\subsection{The absorbing property of the process}

Theorem 3 Assume that the Bayes replacement policy stated in Theorem 2 is adopted. Then, the process $\{X(t), t \geq 0\}$ has two absorbing states 0 and $n$, i.e. the system will not change its state as soon as all the components in use are of the same type. 
Proof: Let $t_{0}=0<t_{1}<\cdots<t_{l}<\cdots$, be the successive failure times of the process and $X_{l}=n_{1 l+1}$. Suppose, without loss of generality, that stage $l$ is the first time when all the components in use are of the same type, type 0 , say, for explicitness, i.e.

$$
X_{l}=0 ; \quad 0<X_{j}<n, \quad 0 \leq j<l .
$$

Then, obviously, $X_{l-1}=1, I_{l}=1, R_{l}=0$ and $I_{l+1}=0$ since all the components in use are of type 0 . Furthermore,

$$
d_{l+1}=x^{2 I_{l+1}-1} \frac{n_{1 l+1}+x n_{0 l+1}}{x n_{1 l+1}+n_{0 l+1}} d_{l}=d_{l},
$$

which implies that $R_{l+1}=R_{l}=0$. Hence, $X_{l+1}=0$.

The proof can be completed by standard induction.

\subsection{Simulation of the process}

Consider the system discussed in Section 3 with the assumption that $\Lambda=\left(\Lambda_{0}, \Lambda_{1}\right)$ has two possible values $(u, v)$ and $(v, u)$ with $u<v$ and $\pi(u, v)=\pi(v, u)=1 / 2$. Let $X(t)$ denote the number of components of type 1 , operating in the system at time $t$. Let $t_{0}=0<t_{1}<\cdots<t_{l}<\cdots$ be the successive failure times and $X_{l}=n_{1 l+1}, l \geq 0$, the number of components of type 1 , operating in the system between the $l$ th and $(l+1)$ th failure. Then, the discrete time stochastic process $\left\{X_{l}, l \geq 0\right\}$ may be simulated by using computer experiments. The initialization step and the main steps are as follows.

Step 0. Let $x_{0}=m=n / 2$ and fix the ratio of $\Delta=\lambda_{0} / \lambda_{1}>1$, say. Let $l=0$, $d_{0}=1$ and go to Step 1 .

Step 1. Generate $I_{l+1}$, which may be 0 or 1 , by using random numbers such that

$$
P\left\{I_{l+1}=1 \mid X_{l}=x_{l}\right\}=\frac{x_{l} \lambda_{1}}{x_{l} \lambda_{1}+\left(n-x_{l}\right) \lambda_{0}}=\frac{x_{l}}{x_{l}+\left(n-x_{l}\right) \Delta},
$$

and go to Step 2.

Step 2. Calculate $d_{l+1}$ using

$$
d_{l+1}=\Delta^{1-2 I_{l+1}} \frac{x_{l} \Delta+\left(n-x_{l}\right)}{x_{l}+\left(n-x_{l}\right) \Delta} d_{l},
$$

and go to Step 3.

Step 3. Determine $R_{l+1}$ by

$$
R_{l+1}=1(0), \text { if } d_{l+1}>(<) 1,
$$

and go to Step 4.

Step 4. Evaluate $x_{l+1}$ by 


$$
x_{l+1}=x_{l}+R_{l+1}-I_{l+1},
$$

and go to Step 5.

Step 5. Decide whether to continue or to stop: if $0<x_{l+1}<n$, then replace $l$ by $l+1$ and go back to Step 1 ; otherwise, if $x_{l+1}=0$ or $n$, then stop.

By using a supercomputer to simulate the process $\left\{X_{l}, l \geq 0\right\}$, we can estimate the relevant quantities of the process we are interested in. For example, we may estimate the probability of absorption into the better of the two absorbing states, the mean time to absorption, and the average proportion of the retrograde or forward motion. (See next section) The results of computer experiments simulating $10^{5}$ paths each for $n=4,8,12, \Delta=1.5,2.00,2.50,3.00,4.00$ are listed in Table 2 through Table 4 .

Table 2. Absorption Probabilities $(\mathcal{N})$

\begin{tabular}{|r|ccccc|}
\hline$\Delta$ & 1.50 & 2.00 & 2.50 & 3.00 & 4.00 \\
\hline $\mathrm{n}=4$ & 0.719 & 0.830 & 0.888 & 0.922 & 0.957 \\
\hline 8 & 0.816 & 0.928 & 0.967 & 0.983 & 0.994 \\
\hline 12 & 0.876 & 0.970 & 0.990 & 0.996 & 0.999 \\
\hline
\end{tabular}

Table 3. Mean Time to Absorption $(\mathcal{N})$

\begin{tabular}{|r|rrrrr|}
\hline$\Delta$ & 1.50 & 2.00 & 2.50 & 3.00 & 4.00 \\
\hline $\mathrm{n}=4$ & 5.84 & 5.18 & 4.66 & 4.25 & 3.70 \\
\hline 8 & 17.7 & 13.7 & 11.6 & 10.0 & 8.31 \\
\hline 12 & 30.4 & 22.8 & 18.3 & 15.8 & 12.9 \\
\hline
\end{tabular}

Table 4. Average Forward Proportion $(\mathcal{N})$

\begin{tabular}{|r|ccccc|}
\hline$\Delta$ & 1.50 & 2.00 & 2.50 & 3.00 & 4.00 \\
\hline $\mathrm{n}=4$ & 0.638 & 0.723 & 0.776 & 0.814 & 0.862 \\
\hline 8 & 0.706 & 0.810 & 0.859 & 0.889 & 0.921 \\
\hline 12 & 0.761 & 0.861 & 0.903 & 0.923 & 0.946 \\
\hline
\end{tabular}

Also, for the purpose of comparison, Table 5 through Table 7 include the corresponding results for the Markov chains under the following replacement policies: the $\varepsilon$-optimal replacement policy $\mathcal{P}_{0.1}$ and the Play the Winner policy $\mathcal{P}$ (see Section 2.3). The results for the non-Markov chain discussed above are listed in the columns under the letter $\mathcal{N}$.

\subsection{The retrograde motions}

To further compare the Bayes replacement policy derived above with the $\varepsilon$-optimal policies studied in Section 2, let us investigate the mean time to absorption and 
Table 5. Absorption Probabilities

\begin{tabular}{|c|ccc|ccc|}
\hline \multirow{3}{*}{$\Delta$} & \multicolumn{3}{|c|}{$\mathrm{n}=4$} & \multicolumn{3}{c|}{$\mathrm{n}=8$} \\
\cline { 2 - 7 } & $\mathcal{P}_{0.1}$ & $\mathcal{P}$ & $\mathcal{N}$ & $\mathcal{P}_{0.1}$ & $\mathcal{P}$ & $\mathcal{N}$ \\
\hline 1.5 & 0.770 & 0.734 & 0.718 & 0.945 & 0.929 & 0.815 \\
\hline 2.0 & 0.884 & 0.849 & 0.830 & 0.992 & 0.988 & 0.928 \\
\hline 3.0 & 0.960 & 0.939 & 0.921 & 0.999 & 0.998 & 0.982 \\
\hline 4.0 & 0.985 & 0.969 & 0.957 & 0.999 & 0.999 & 0.993 \\
\hline
\end{tabular}

Table 6. Mean Time to Absorption

\begin{tabular}{|c|ccc|ccc|}
\hline \multirow{3}{*}{$\Delta$} & \multicolumn{3}{|c|}{$\mathrm{n}=4$} & \multicolumn{3}{c|}{$\mathrm{n}=8$} \\
\cline { 2 - 7 } & $\mathcal{P}_{0.1}$ & $\mathcal{P}$ & $\mathcal{N}$ & $\mathcal{P}_{0.1}$ & $\mathcal{P}$ & $\mathcal{N}$ \\
\hline 1.5 & 64.41 & 7.32 & 5.84 & 709.3 & 85.3 & 17.7 \\
\hline 2.0 & 52.06 & 6.30 & 5.18 & 349.7 & 46.1 & 13.7 \\
\hline 3.0 & 37.45 & 4.93 & 4.25 & 148.8 & 22.7 & 10.0 \\
\hline 4.0 & 29.90 & 4.20 & 3.70 & 95.5 & 15.5 & 8.31 \\
\hline
\end{tabular}

Table 7. Average Forward Proportion

\begin{tabular}{|c|ccc|ccc|}
\hline \multirow{3}{*}{$\Delta$} & \multicolumn{3}{|c|}{$\mathrm{n}=4$} & \multicolumn{3}{c|}{$\mathrm{n}=8$} \\
\cline { 2 - 7 } & $\mathcal{P}_{0.1}$ & $\mathcal{P}$ & $\mathcal{N}$ & $\mathcal{P}_{0.1}$ & $\mathcal{P}$ & $\mathcal{N}$ \\
\hline 1.5 & .508 & .614 & .638 & .506 & .556 & .706 \\
\hline 2.0 & .518 & .688 & .723 & .509 & .597 & .810 \\
\hline 3.0 & .532 & .768 & .814 & .512 & .659 & .889 \\
\hline 4.0 & .545 & .815 & .862 & .512 & .706 & .921 \\
\hline
\end{tabular}

the average proportion of correct and wrong replacements for each policy. Assume, from now on, that the components of type 1 are better. Then, at each failure, a new component of type 1 should be the right choice for the replacement. This lets the Markov process move forward to the better absorbing state $n$. On the other hand, a component of type 0 is a wrong choice for the replacement, which leads to a retrograde motion towards the inferior absorbing state 0 .

For a given replacement policy and the resulting stochastic process, let the random variable $T$ be the total number of replacements until absorption. Then, $R=\sum_{l=1}^{T} R_{l}$ and $W=T-R$ are the number of right and wrong replacements, which are made by components of type 1 and 0 , respectively. Let the ratio of $B=W / T(R / T)$ be called the retrograde (forward) proportion of the policy and its expectation $E(B)(E(R / T))$ the average retrograde (forward) proportion of the policy.

Since no closed-form representations are available, computer simulations are employed to approximate $E(T)$ and $E(B)$. The results are given in Table 6 and 7 .

From Table 5 one can see that the $\varepsilon$-optimal policies for the absorbing Markov processes possess greater probabilities of absorption into the better terminal state. 
On the other hand, from Table 6 and Table 7, the Bayes replacement policy derived above requires shorter duration of time for the process to get absorbed, has much greater average proportion of forward motions, and hence, much less inferior components to be used in the course of time.

\section{Acknowledgements}

The author thanks the Managing Editor of JAMDS and two anonymous referees for their helpful comments and suggestions, which improved the presentation of the paper. Special thanks go to Professor K. J. Miescke for his helpful comments and encouragements. Research support from Indiana University Southeast is gratefully acknowledged.

\section{References}

1. K. Alam. Selection from Poisson processes. Ann. Inst. Statist. Math., 23:411-418, 1971.

2. K. Alam and J. Thompson. A problem of ranking and estimation with Poisson processes. Technometrics, 15:801-808, 1973.

3. D. Dixon and R. Bland. A Bayes solution to the problem of ranking Poisson parameters. Ann. Inst. Statist. Math. , 23:119-124, 1971.

4. P.S. Goel. A note on the non-existence of subset selection procedures for Poisson populations. Mimeograph series no. 303, Department of Statistics, Purdue University, West Lafayette, IN, 1972.

5. S. Gupta and D. Huang. On subset selection procedures for Poisson populations and some applications to the multinomial selection problems. In Applied Statistics, ed. R. Gupta, pages 97-109. North-Holland, Amsterdam, 1975.

6. S. Gupta, Y.K. Leong and W.Y. Wong. On subset selection procedures for Poisson populations. Bull. Malaysian Math Soc. , 2:89-110, 1979.

7. S. Gupta and S. Panchapakesan. Multiple Decision Procedures. John Wiley, New York, 1979.

8. S. Gupta and W. Wong. On subset selection procedures for Poisson processes and some applications to the binomial and multinomial problems. In Operation Research Verfahren Vol. 23 , ed. R. H. et al, pages 49-70. Anton Hain, Meisenheim am Glan, 1977.

9. K. Miescke. Optimum replacement policies for using the most reliable components. Journal of Statistical Planning and Inference, 26: 267-276, 1990.

10. K. Miescke and D. Shi. Optimum Markov replacement policies for using the most reliable components. Journal of Statistical Planning and Inference , 45: 331-346, 1995.

11. D. Shi. Optimum Replacement Policies for Selecting the Best Components.. Doctoral dissertation, the University of Illinois, Chicago, 1993.

12. D. Shi. A dynamic approach for selecting the most reliable components. In Optimizations: Techniques and Applications, Vol. 2: 961-970, 1995.

13. J. Wilson. Robust Bayesian selection of the best Poisson population. In E. Bofinger, E.J. Dudewicz, G.J. Lewis, K. Mengersen, Eds., The Frontiers of Modern Statistical Inference Procedures, II. American Science Press, Columbus, OH, 289-302, 1992. 


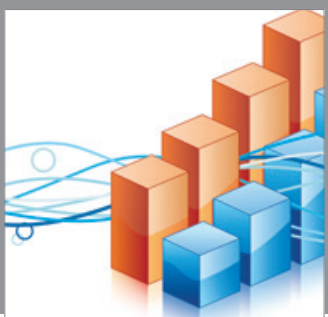

Advances in

Operations Research

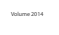

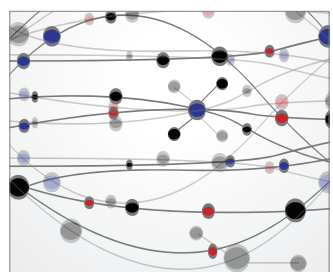

\section{The Scientific} World Journal
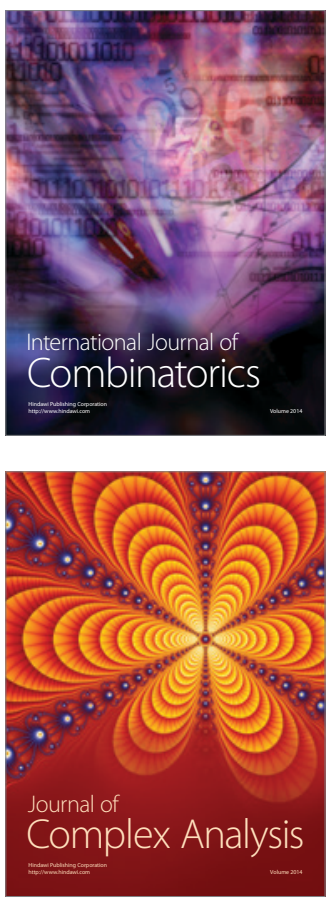

International Journal of

Mathematics and

Mathematical

Sciences
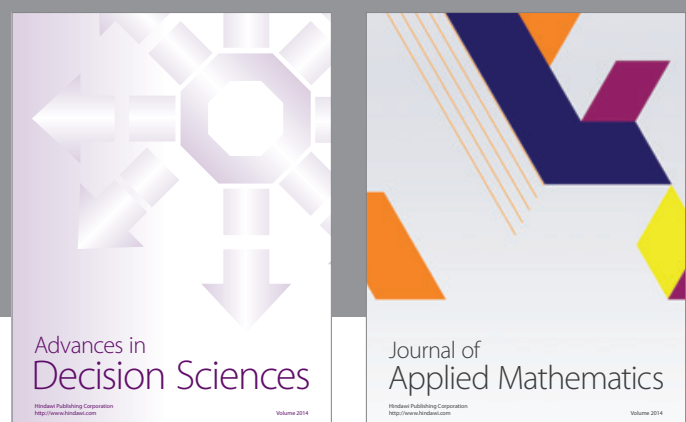

Journal of

Applied Mathematics
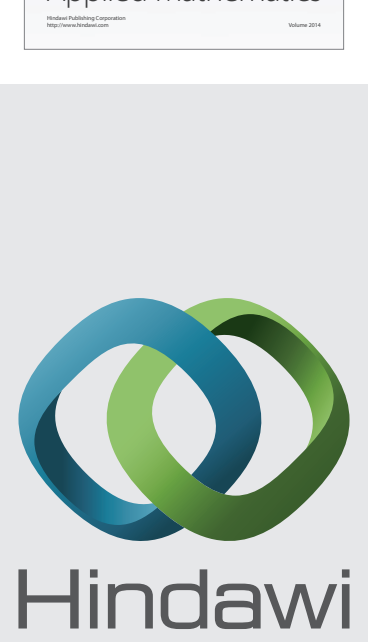

Submit your manuscripts at http://www.hindawi.com
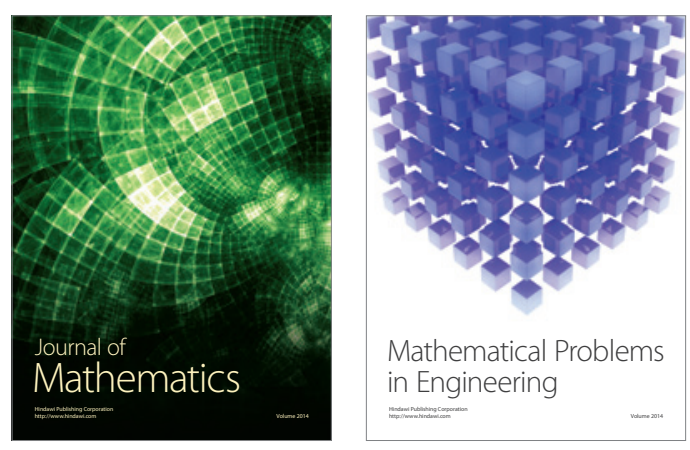

Mathematical Problems in Engineering
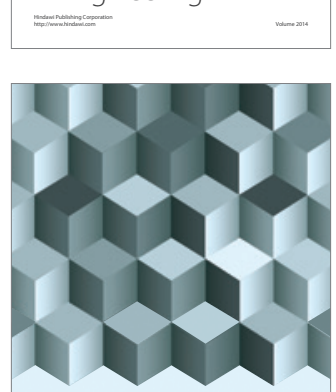

Journal of

Function Spaces
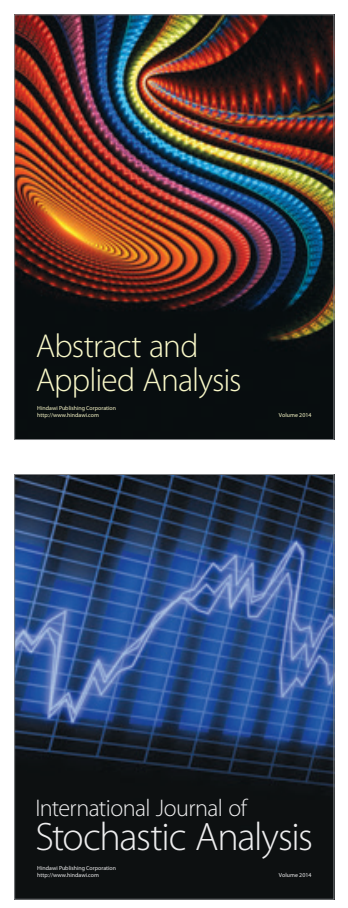

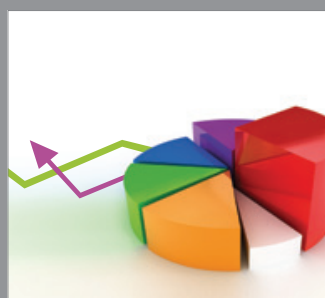

ournal of

Probability and Statistics

Promensencen
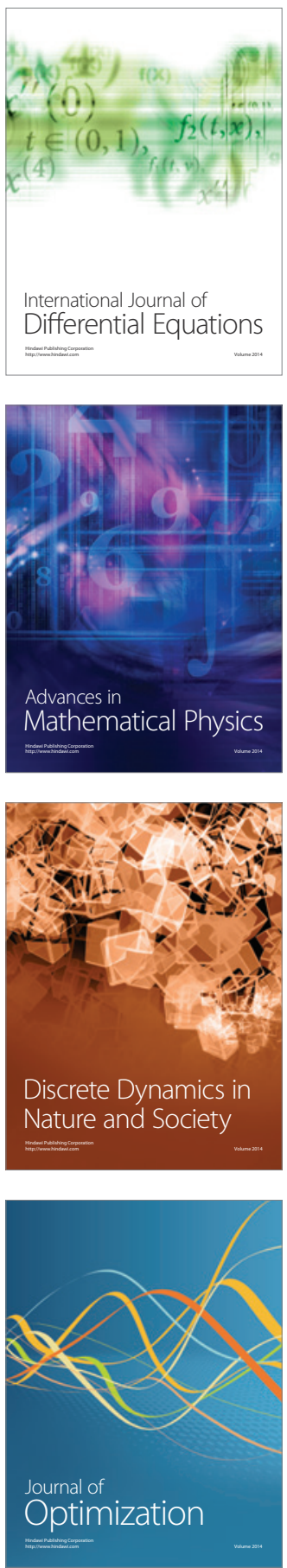